\title{
Management of Ventilator-associated Pneumonia
}

\author{
M. Ferrer, M. Valencia, and A. Torres
}

\section{Introduction}

Pneumonia is the most important respiratory infection in mechanically ventilated patients. It is defined as the presence of microorganisms in the pulmonary parenchyma leading to the development of an inflammatory response by the host, which may be localized in the lung or may extend systemically. Nosocomial pneumonia is an infectious process which develops within 48 hours after admission to the hospital and that was not incubating at the time of hospitalization. Ventilator-associated pneumonia (VAP) is considered as a subgroup of nosocomial pneumonia and is an infectious pulmonary process which develops 48 hours after the presence of an artificial airway and mechanical ventilation. Since a large proportion of the patients who develop nosocomial pneumonia are intubated and receive mechanical ventilation, most epidemiological and clinical studies on nosocomial pneumonia have been focused on critically ill patients and those receiving mechanical ventilation. From a clinical point of view, nosocomial pneumonia is of great importance not only because of the consequences of the important morbidity and mortality but also due to the high costs associated with development of this disease.

\section{Epidemiology}

Despite the large amount of data available on the epidemiology of VAP, the results provided by the different studies vary widely. This may be due to the lack of a standardized diagnostic approach and the different populations studied. Moreover, although VAP has been well defined, disagreement as to the final diagnosis may be attributed to: 1) focal areas of the lobe which may be missed;2) negative microbiological studies despite the presence of inflammation in the lung; and 3) pathologists may disagree in their conclusions.

The point of time at which VAP develops has important implications in the etiology, treatment and diagnosis of this disease. VAP has classically been determined as early-onset pneumonia, which occurs within the first four days after hospital admission, and late-onset pneumonia, which develops five or more days after admission [1].

Nosocomial pneumonia is the second most common hospital-acquired infection and is the leading cause of death among this type of infection. The incidence of nosocomial pneumonia ranges from 4 to 50 cases per 1000 admissions in community hospitals and general medical wards to up to 120 to 220 cases per 1000 admissions in some intensive care units (ICUs) or among patients requiring mechanical ventila- 
tion. The European Prevalence of Infection in Intensive Care (EPIC) study [2], a large 1-day point prevalence study of infections, was carried out in 1417 ICUs and included 10,038 patients. The prevalence of ICU-acquired infections was of $21 \%$; $47 \%$ of these patients had pneumonia, of nosocomial origin in $10 \%$. In a large, prospective cohort study including 1014 patients receiving mechanical ventilation, 177 (18\%) developed VAP [3]. The incidence of this disease was $24 \%(78 / 322)$ in a Spanish study on risk factors for VAP [4]. In a recently published study, the mean incidence of hospital-acquired pneumonia in non-ICU patients was $3 \pm 1.4$ cases/ 1000 hospital admissions [5]. Most patients were in medical wards (64\%), had severe underlying diseases, and the hospital stay was greater than 5 days.

The overall incidence of VAP varies from $8-28 \%$. A prospective Italian study on VAP including 724 critically ill patients who had received prolonged ventilatory support after admission reported an incidence of $23 \%$ [6]. This rate rose from $5 \%$ in patients receiving mechanical ventilation for 1 day to $69 \%$ in those receiving mechanical ventilation for more than 30 days. In a study including 567 patients receiving mechanical ventilation, evaluated with invasive procedures, the rate of VAP was $9 \%$ [7]. In this latter study the cumulative risk of pneumonia was estimated to be $7 \%$ at 10 days and $19 \%$ at 20 days after initiation of mechanical ventilation, thereby showing the classical incremental risk of pneumonia of $1 \%$ per day [7]. However, in a large series of 1014 patients receiving mechanical ventilation, Cook et al. [8] described a rate of VAP of $18 \%$, and although the cumulative risk for developing VAP increased over time, the daily hazard rate decreased after day 5 . The risk per day was determined to be $3 \%$ on day $5,2 \%$ on day 10 , and $1 \%$ on day 15 .

Several studies have reported the rate of mortality in VAP to range from $24-76 \%$ $[4,7,9]$. Patients with VAP in the ICU receiving mechanical ventilation may have a 2 to 10 -fold greater risk of death compared to patients without this complication. In a study of 78 episodes of nosocomial pneumonia detected in 322 consecutive patients receiving mechanical ventilation, the overall mortality rate was $23 \%$ [4]. Similarly, the Neunos 2000 study group reported a mortality of $26 \%$ (pneumoniaattributed $13.9 \%$ ) in 186 non-ICU patients with hospital-acquired pneumonia [5]. The mortality of patients with nosocomial pneumonia was higher $(33 \%)$ when compared with rates of patients without nosocomial pneumonia $(19 \%, \mathrm{p}<0.01)$. On step-forward logistic regression analysis the identification of 'high-risk' microorganisms (Pseudomonas aeruginosa, Enterobacteriaceae, and other Gram-negative bacilli, Enterococcus faecalis, Staphylococcus aureus, Candida spp. Aspergillus spp, and episodes of polymicrobial pneumonia), bilateral involvement on chest X-ray, the presence of respiratory failure, inappropriate antibiotic therapy, age over 60 years, or an ultimately or rapidly fatal underlying condition were independently associated with worse prognosis.

The increased risk ratios of mortality in patients with VAP vary from 1.7 to 4.4 [10]. Although several studies have shown that VAP is a severe disease, the controversy as to attributing mortality to nosocomial pneumonia continues. However, several studies have shown nosocomial pneumonia to be an independent prognostic factor. Patients in whom the attributable mortality is increased include patients undergoing cardiac surgery, patients with acute lung injury, and immunosuppressed patients [11]. In contrast, in patients with life-threatening medical conditions such as cardiac arrest in young patients with no underlying disease and those admitted due to trauma, nosocomial pneumonia does not seem to significantly increase mortality [12]. 


\section{Pathogenesis}

In developing pneumonia, virulent microorganisms must invade the lung parenchyma. This may occur either as the result of a defect in defense mechanisms of the host or by an overwhelming inoculum. The normal human respiratory tract has a variety of defense mechanisms such as anatomic barriers, cough reflex, cell and humoral-mediated immunity and a dual phagocytic system involving both alveolar macrophages and neutrophils. Virulent microorganisms can reach the alveolar space in several ways, such as colonization of the upper airway by potentially pathogenic microorganisms and posterior microaspiration, macroaspiration of gastric contents, contaminated respiratory care equipment such as condensates in ventilator tubing, fiberoptic bronchoscopes, tracheal suctioning material, or nebulizers, the hematogenous route and direct dissemination from contiguous sites such as the pleura, the pericardium or the abdomen.

Oropharyngeal and tracheal colonization play a central role in the pathogenesis of VAP. Early colonization (within the first 24 hours of mechanical ventilation) has been described in patients who are intubated and in those receiving mechanical ventilation, varying from $80 \%$ to $89 \%$ [13]. One study [14] demonstrated that $45 \%$ of 213 patients admitted to a medical ICU became colonized with aerobic Gram-negative bacilli by the end of one week in the hospital. Among the 95 colonized patients, $23 \%$ developed nosocomial pneumonia while only four out of the 118 non-colonized patients developed pneumonia. Among the microorganisms colonizing the trachea, Pseudomonas spp. has an increased affinity to ciliated tracheal epithelial cells and these microorganisms are not usually present in the oropharynx. Adherence of Pseudomonas increases in desquamated epithelium following influenza virus infection, tracheostomy, or repeated tracheal suctions in intubated patients [15]. In a study including 86 patients receiving mechanical ventilation, oropharyngeal colonization, which was detected either on admission or from subsequent samples, was a predominant factor of nosocomial pneumonia compared with gastric colonization [16]. Oropharyngeal colonization with Acinetobactor baumanii yielded an estimated 7.45-fold increased risk of pneumonia compared with patients who had not yet or who were not identically colonized $(p=0.0004)$. DNA genomic analysis demonstrated that an identical strain was isolated from oropharyngeal or gastric samples and bronchial samples in all but three cases of pneumonia due to $S$. aureus [16].

\section{Risk Factors}

There are considerable amounts of data concerning risk factors for VAP. These factors are important since they may contribute to the development of effective prevention programs by indicating which patients may be most likely to benefit from prophylaxis against pneumonia. We herewith discuss the most relevant risk factors for

Table 1. Risk factors for nosocomial pneumonia.

\begin{tabular}{ll}
\hline Reintubation & Supine position \\
Decrease in pressure of the tracheal tube cuff & Coma and head trauma \\
Stress-ulcer prophylaxis $\left(a n t i-\mathrm{H}_{2}\right)$ & Nasogastric tube and gastric distension \\
Tracheostomy & Patient transport \\
\hline
\end{tabular}


endogenous infection in VAP, although data on many of these factors continue to be controversial. These risk factors are listed in Table 1.

\section{Antimicrobial Agents}

The distribution of microorganisms, especially potentially-resistant bacteria, differs in patients who have or have not received prior antibiotic therapy. Likewise, previous antibiotic administration may influence the development of VAP in two different ways: Its use may be associated with a protective effect against early-onset pneumonia, while, on the other hand, it may be associated with an increased risk of lateonset pneumonia. To determine the baseline and time-dependent risk factors for VAP, Cook et al. [8] evaluated 1014 patients. On multivariate analysis, independent predictors of VAP were a primary diagnosis on admission of burns (risk ratio 5.09), trauma (risk ratio 5.00), central nervous system disease (risk ratio 3.40), respiratory disease, cardiac disease (risk ratio 2.72), mechanical ventilation within the previous 24 hours (risk ratio 2.28), witnessed aspiration (risk ratio 3.25) and paralytic agents. Exposure to antibiotics conferred protection (risk ratio 0.37), but this effect attenuated over time. Rello et al. [17] evaluated the risk factors for VAP within the first 8 days of mechanical ventilation in 83 consecutive intubated patients undergoing continuous aspiration of subglottic secretions. Multivariate analysis showed the protective effect of antibiotic use (relative risk 0.10 ) whereas failure of continuous aspiration of subglottic secretions (relative risk 5.29) was associated with a greater risk of pneumonia. In addition, Sirvent et al. [18] evaluated the use of systemic prophylaxis with cefuroxime before intubation on the incidence of VAP in 100 patients with coma, 50 of whom received one dose of $1.5 \mathrm{~g}$ of cefuroxime intravenously at the time of intubation and a second dose $12 \mathrm{~h}$ later. The global incidence of early-onset VAP was $37 \%(n=37): 12(24 \%)$ in the cefuroxime group and $25(50 \%)$ in the control group $(p=0.007)$. All these studies demonstrate the protective effect of antibiotic therapy in early VAP caused by endogenous flora. In a prospective study in 277 patients receiving mechanical ventilation, Kollef [10] determined that the following four factors were associated with VAP: Index of systemic organ failure $\geq 3$ (odds ratio $[O R]=10.2)$, age $\geq 60$ years $(O R=5.1)$, previous antibiotics $(O R=3.1)$, and supine head position within the first 24 hours of mechanical ventilation $(\mathrm{OR}=2.9)$. In addition to the importance of antibiotic therapy as a risk factor for VAP, the influence of antibiotics on the etiology of this disease is also relevant. In a prospective study, Rello et al. [19] studied 129 consecutive episodes of VAP to evaluate the influence of prior antibiotic administration on the etiology and mortality of VAP. The rate of VAP caused by Gram-positive cocci or Haemophilus influenzae was statistically lower $(p<0.05)$ in patients who had received antibiotics previously while the rate of VAP caused by $P$. aeruginosa was statistically higher $(\mathrm{p}<0.01)$. Step-forward logistic regression analysis only determined previous antibiotic use (OR 9.2) as significantly influencing the risk of death in VAP. Likewise, Trouillet and coworkers [20] demonstrated that three variables remained significantly associated with potentially resistant microorganisms as a causative etiology in VAP: Duration of mechanical ventilation $\geq 7$ days (OR 6.0), prior antibiotic use (OR 13.5), and prior use of broad-spectrum drugs (third generation cephalosporin, fluoroquinolones, and/or imipenem) (OR 4.1). All of these studies have demonstrated that antimicrobial therapy has a bimodal effect on the development of VAP. Antibiotics protect against early-onset pneumonia, especially pneumonia caused by endogenous flora, but they are also responsible for the selection of resistant microorganisms causing 
late-onset pneumonia such as $P$. aeruginosa and methicillin-resistant $S$. aureus (MRSA).

\section{Body Position}

It has been demonstrated that up to $50 \%$ of healthy adults aspirate at night. However, in these subjects it is not clinically significant since lung defense mechanisms remain intact. Torres and coworkers $[21,22]$ demonstrated the importance of body position in gastroesophageal reflux and tracheal aspiration. These authors instilled a colloid with technetium via the nasogastric tube, and by placing patients in a semirecumbent position they found a significant reduction in the radioactivity of tracheal secretions compared with patients in the supine position. Moreover, in another randomized study [23], this group studied the impact of body position on the development of VAP. Patients were placed in a semirecumbent $\left(45^{\circ}\right)$ or supine $\left(0^{\circ}\right)$ body position. Microbiologically-confirmed pneumonia developed in $5 \%$ of the patients in the semirecumbent position and in $23 \%$ of those in the supine $(p=0.018)$.

\section{Gastric Colonization and Stress Ulcer Prophylaxis}

Low gastric pH prevents against bacterial growth in the gastric chamber and bacterial migration from the small bowel. The relationship between gastric $\mathrm{pH}$ and gastric colonization has been well established in several studies. The use of prophylactic agents for stress ulcers, which alter the gastric $\mathrm{pH}$, may increase gastric colonization and the rates of VAP, although this remains to be demonstrated. In a 1991 metaanalysis, Tryba [24] found that antacids and $\mathrm{H}_{2}$-antagonists were significantly more effective in preventing stress bleeding in treated versus untreated patients. Sucralfate was superior to $\mathrm{H}_{2}$-antagonists. Patients treated with antacids or $\mathrm{H}_{2}$-antagonists showed a significantly higher risk for the development of nosocomial pneumonia. In a later study, Cook et al. [25] demonstrated a trend toward less clinically important bleeding with $\mathrm{H}_{2}$-antagonists and antacids than with sucralfate. They found a trend toward an increased risk of pneumonia associated with $\mathrm{H}_{2}$-antagonists compared with no prophylaxis and a significantly higher risk compared to sucralfate. Finally, another meta-analysis [26] concluded that ranitidine is not effective in the prevention of gastrointestinal bleeding and may increase the risk of pneumonia. Studies on sucralfate do not provide conclusive results. Currently, there are not enough data to give a conclusive recommendation.

\section{Diagnosis}

The first problem in the diagnosis of nosocomial pneumonia and VAP is the lack of a gold-standard for comparing the different techniques used to confirm suspicion of an infectious process. Despite the use of histology in pulmonary biopsy and cultures of pulmonary tissue in the immediate post-mortem period, the value of these techniques in parenchymal infections has not been unequivocally demonstrated. Suspicion of nosocomial pneumonia depends on the finding of new and persistent infiltrates on chest X-ray in association with some clinical signs and symptoms (fever or hypothermia, purulent respiratory secretions and leukocytosis or leukopenia). Based on histology and microbiological cultures of post-mortem pulmonary biopsies, one 
Table 2.The Clinical Pulmonary Infection Score [28]

\begin{tabular}{|c|c|c|c|}
\hline Criterion & 0 & 1 & 2 \\
\hline $\begin{array}{l}\text { Tracheal secretions } \\
\text { Pulmonary Radiology } \\
\text { Temperature, }{ }^{\circ} \mathrm{C} \\
\mathrm{Blood} \text { leukocytes } \\
\mathrm{PaO}_{2} / \mathrm{FiO}_{2} \\
\mathrm{Microbiology}^{*}\end{array}$ & $\begin{array}{c}\text { Absent } \\
\text { No } \\
\geq 36.5 \text { and } \leq 38.4 \\
\geq 4000 \text { and } \leq 11000 \\
>240 \text { or ARDS } \\
\text { Negative }\end{array}$ & $\begin{array}{c}\text { Non-purulent } \\
\text { Diffuse } \\
\geq 38.5 \text { and } \leq 38.9 \\
<4000 \text { or }>11000\end{array}$ & $\begin{array}{c}\text { Purulent } \\
\text { Localized } \\
\geq 39 \text { or } \leq 36 \\
=+ \text { bands }>50 \% \\
\leq 240 \text { without ARDS } \\
\text { Positive }\end{array}$ \\
\hline
\end{tabular}

* Culture evaluation delay at least $24 \mathrm{~h}$; ARDS: acute respiratory distress syndrome

study demonstrated that the presence of radiological findings plus two or more clinical criteria showed a sensitivity and specificity of $69 \%$ and $75 \%$, respectively [27].

In recent years the Clinical Pulmonary Infection Score (CPIS), validated by Pugin and coworkers [28], has been widely used. This score combines different clinical, radiological, physiological, laboratory and microbiological parameters (Table 2) in order to increase the specificity of the clinical diagnostic approach. A score greater than 6 demonstrates a good correlation with the presence of pneumonia. The results of different studies on the diagnostic performance of this score are contradictory with values of sensitivity and specificity of around $77 \%$ and $42 \%$, respectively [27]. Some studies have been aimed at increasing the diagnostic yield of this score with the addition of Gram staining of secretions of the lower respiratory tract [29].

The diagnostic tests performed on suspicion of VAP have two objectives: The first is to determine whether the patient really has an infectious pulmonary process as indicated by the signs and symptoms leading to the use of these tests; the second is the isolation of the causative microorganisms of the disease.

For many years, the diagnostic performance of the different techniques used to confirm suspicion of VAP has been under debate. At present, the value of non-invasive tests, such as tracheobronchial aspirate, and invasive bronchoscopic tests, such as bronchoalveolar lavage (BAL) and the protected specimen brush (PSB), as well as the advantages and disadvantages of each technique have been well established [30]. As a general rule, tracheobronchial aspirate has very good sensitivity with a specificity a little lower than the invasive tests when using a quantitative culture of the respiratory secretions obtained by this method [31]. In different studies, the sensitivity of this test varies from $38-100 \%$ with a specificity of $14-100 \%$ [32]. A negative tracheobronchial aspirate culture in a patient who has not received antibiotic treatment has a high negative predictive value for the presence of VAP. In one study on the diagnostic value of this technique, the negative predictive value was $72 \%$ in 102 patients evaluated with this technique and with invasive methods. The sensitivity of PSB and BAL are $33-100 \%$ and $42-93 \%$, respectively and the specificities are $50-100 \%$ and $45-100 \%$ [7]. On the other hand, the current controversy lays in the role invasive and non-invasive methods have in the prognosis and use of antibiotics in these patients. On analyzing only the randomized studies, we found that in a pilot study with 51 patients Sánchez-Nieto and coworkers [33] observed that bronchoscopic methods led to a greater change in initial antibiotic treatment ( $42 \%$ versus $16 \%, \mathrm{p}<0.05$ ) with no significant differences as regards to either global or attributable mortality or morbidity. This study was limited by its small sample size and the lack of a standard treatment protocol in the invasive group. The study by Solé-Violan and coworkers [34] demonstrated a greater number of antibiotic changes with invasive techniques with no clear influence on mortality, length of ICU stay, and 
days on mechanical ventilation. Ruiz and coworkers [35] compared 765 patients with suspicion of VAP ( 39 non-invasive and 37 invasive) and concluded that the diagnostic performance of both techniques in VAP was similar, as was mortality at 30 days, number of days on mechanical ventilation, and length of ICU stay. The costs of invasive studies were clearly greater. One study by Fagon and coworkers [36] reported positive results with respect to a decrease in mortality on day 14 and

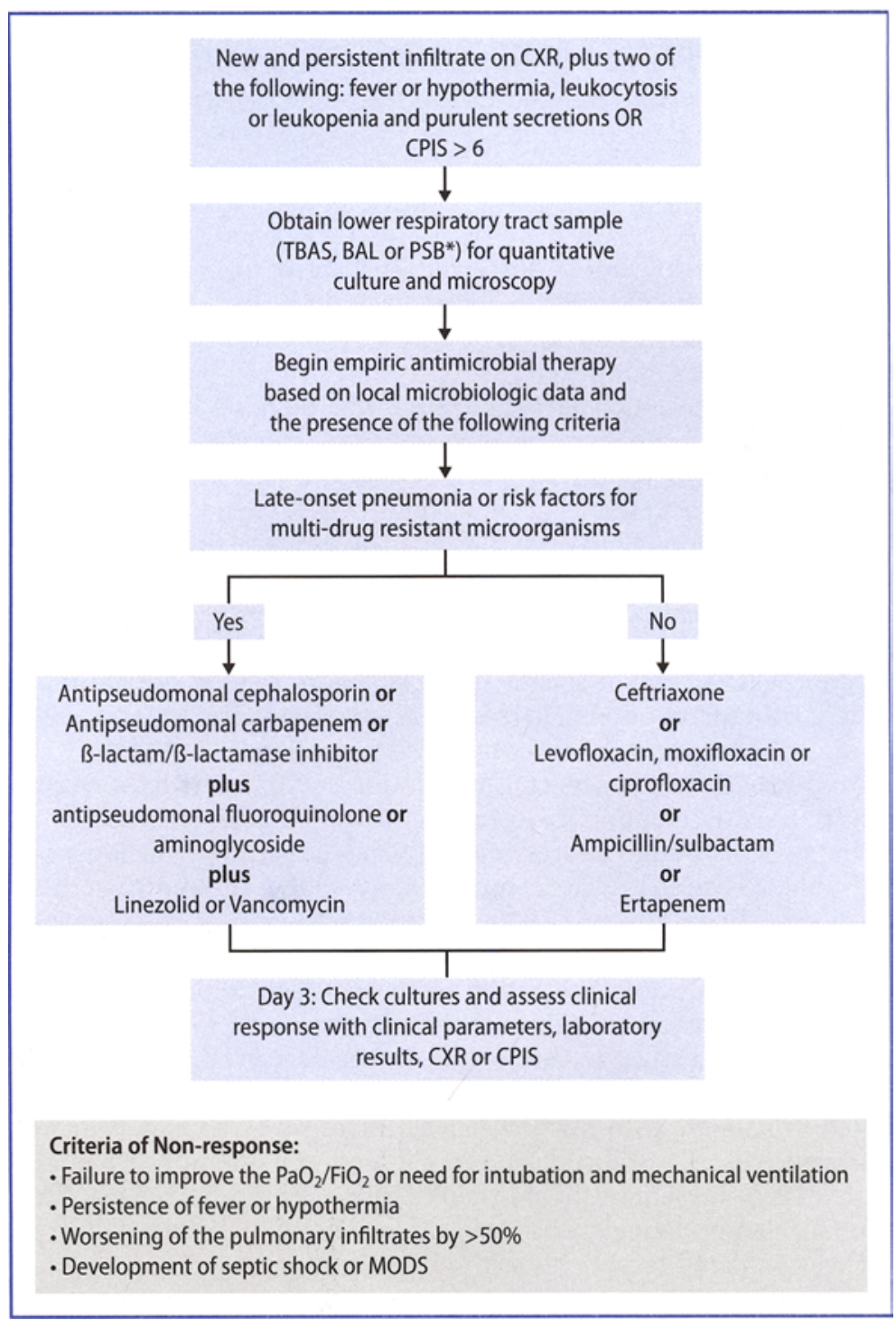

Fig. 1. Algorithm for the management of patients with nosocomial pneumonia. CXR: Chest $X$ Ray; CPIS: Clinical Pulmonary Infection Score; TBAS: Tracheobronchial aspirate; BAL: Bronchoalveolar lavage; PSB: Protected specimen brush; $\mathrm{PaO}_{2}$ : Oxygen arterial pressure; $\mathrm{FiO}_{2}$ : Inspired fraction of oxygen; MODS; Multiple organ dysfunction syndrome 
Sequential Organ Failure Assessment (SOFA) scores on days 3 and 7, a reduction in the use of antibiotics, and the number of antibiotic-free days with the invasive technique. Nonetheless, the study was limited by the use of qualitative cultures of the tracheal aspirates which thereby limited comparison with the other studies.

In summary, we suggest the following diagnostic and management approach to nosocomial pneumonia (Fig. 1). First, clinical suspicion of pneumonia should be based on classical clinical criteria or a CPIS $>6$. Respiratory secretions should be collected at this time by tracheobronchial aspirate or bronchoscopy for obtaining quantitative cultures. Reevaluation should be made at 48 to $72 \mathrm{~h}$ and the decision as to whether to continue antimicrobial treatment should be based on the probability of pneumonia, the results of the cultures, and the presence of an alternative diagnosis [37].

\section{Treatment}

When deciding to treat a suspected episode of ICU-acquired pneumonia, several aspects should be taken into account. The importance of early initiation of adequate antimicrobial therapy for the treatment of VAP has been emphasized in recent years in several studies. Alvarez-Lerma [38] evaluated 430 patients with VAP and found a higher attributable mortality $(24.7 \%$ vs. $16.3 \%, \mathrm{p}=0.039)$ and a higher incidence of septic shock and gastrointestinal bleeding among patients receiving inadequate initial treatment. Similar results were also reported by Luna and coworkers [39] and Rello and Diaz [40]. With mini-BAL fluid cultures, Kollef and Ward [41] reported that inappropriate antibiotic therapy was associated with an OR for death of 3.28.

Empiric antimicrobial therapy may be inadequate as a consequence of the presence of unexpected pathogens not covered by the initial antibiotic schedule but this may be mainly due to unanticipated resistance. In most of the previously mentioned studies, a large proportion of the episodes of inadequate antimicrobial treatment were attributed to potentially resistant Gram-negative bacteria (especially $P$. aeruginosa, Acinetobacter spp. and Enterobacter spp.) or MRSA.

Another important aspect to take into account is whether modification of the initial inadequate therapy according to microbiological results improves the outcome of the patient. Studies addressing this issue did not find any improvement in mortality with this strategy. Luna and coworkers [39] showed that therapeutic changes made after bronchoscopy led to more patients $(n=42)$ receiving adequate therapy. Nonetheless, the mortality in this group was comparable to the mortality reported among patients who continued to receive inadequate therapy $(n=23)$. In a study by Rello and coworkers [42], bronchoscopic results led to a change in antibiotic treatment in 27 cases $(24 \%)$ considered to have received inadequate initial treatment. Despite clinical resolution in 17 of these cases $(63 \%)$, the mortality was higher compared to patients with initial adequate therapy. Kollef and Ward [41] found a high prevalence $(73 \%)$ of inadequate initial antibiotic therapy in a study of 130 patients with VAP. In this study, the mortality of patients in whom the antibiotic therapy had been started or changed based on the results of mini-BAL culture was significantly higher compared to patients with unchanged or discontinued treatment $(60.8 \%$ vs. $33.3 \%$ and $14.3 \%$, respectively).

The results of these studies demonstrate the need for early initiation of broad-spectrum empirical antimicrobial therapy on suspicion of VAP. The American Thoracic Society (ATS) published its first guidelines for the management of hospital-acquired pneumonia in 1995 [43]. In 1998, Trouillet et al. [20] suggested a different classifica- 
tion for the prediction of pathogens and the selection of antibiotic treatment based on previous antibiotic use and the duration of mechanical ventilation. These classifications provide a different rationale for the prediction of microbial etiology with the aim of aiding clinicians to prescribe appropriate initial empiric therapy.

In a prospective study, we evaluated the level of bacterial coverage and validated the adequacy of the antibiotic strategy proposed by the 1996 ATS guidelines and the Trouillet framework [44]. Both classifications were found to be effective in predicting the pathogen involved ( $91 \%$ and $83 \%$, respectively). However, taking the in vitro sensitivity of the pathogens isolated into account, the adequacy of the antibiotic treatment proposed by these classifications was found to be rather lower $(79 \%$ for ATS and $80 \%$ for Trouillet). The microorganisms involved in treatment inadequacy were multiresistant $P$. aeruginosa, A. baumanii, S. maltophilia, and MRSA. These findings underline the importance of considering additional parameters such as local microbial epidemiology and more accurate models of prediction of resistance to improve the level of coverage and the appropriateness of antibiotic treatment.

The ATS recently published new guidelines for the management of adults with hospital-acquired pneumonia [45] and, contrary to the previous guidelines, the severity of pneumonia does not play an important role in decisions regarding the initial empiric treatment to be implemented (Table 3). Regardless of the severity of pneumonia, patients with risk factors for infection with multi-drug resistant microorganisms or with hospital admission greater than 5 days should receive empiric broad-spectrum antibiotic therapy that adequately covers infection by $P$. aeruginosa. The different schedules recommended for this group include: a cephalosporin with anti-pseudomonal activity, a carbapenem or piperacillin/tazobactam, associated with an aminoglycoside or a fluoroquinolone with antipseudomonal activity. Linezolide or vancomycin should be included in cases with suspicion of MRSA infection or hospitals with a high incidence of this microorganism. Patients who do not fulfil the previously mentioned characteristics should receive empiric treatment with schedules which cover the 'core' microorganisms such as $S$. pneumoniae, $H$. influen$z a e$, methicillin-sensitive $S$. aureus, and antibiotic-sensitive aerobic Gram-negative bacilli. The drugs of choice include ceftriaxone, a fluoroquinolone, a beta-lactam/ beta-lactamase inhibitor, or ertapenem [45].

To date, the use of combined antibiotic therapy is still recommended in the treatment of VAP with suspicion of $P$. aeruginosa or other potentially resistant pathogens. Previous studies on bacteremic infections caused by P. aeruginosa [46] and Klebsi-

Table 3. Likely etiologic pathogens causing nosocomial pneumonia.

\begin{tabular}{ll}
$\begin{array}{l}\text { Patients with no risk factors for MDR pathogens, } \\
\text { early onset, and any severity. }\end{array}$ & $\begin{array}{l}\text { Patients with late-onset pneumonia or risk factors } \\
\text { for MDR pathogens and any severity }\end{array}$ \\
$\begin{array}{ll}\text { Streptococcus pneumoniae } \\
\text { Haemophilus influenzae }\end{array}$ & The same as the previous group \\
$\begin{array}{l}\text { Methicillin-sensitive Staphylococcus aureus (MSSA) } \\
\text { Enteric gram-negative bacilli }\end{array}$ & Plus \\
Escherichia coli & Pseudomonas aeruginosa \\
Klebsiella pneumoniae & Klebsiella pneumoniae (ESBL) \\
Enterobacter spp & Acinetobacter spp \\
Proteus spp & Methicillin-resistant Staphylococcus aureus (MRSA) \\
Serratia marcescens & Legionella pneumophila \\
\hline
\end{tabular}

MDR: Multi-drug resistant; ESBL: Extended spectrum $\beta$-lactamases. 
ella spp. [47] demonstrated a higher mortality associated with the use of initial empiric monotherapy compared to combined therapy. However, these studies are limited in that they were performed when less active beta-lactams were used. Further trials are needed to clarify this issue. For now, the use of monotherapy should be limited to the treatment of severe nosocomial pneumonia in patients without risk factors for potentially resistant pathogens.

The length of antimicrobial treatment is also under debate. In the ATS guidelines [45], the experts recommend that the length of treatment be shortened from the traditional 14-21 days to shorter periods, to 7-10 days. The latter shorter treatment is recommended in the treatment of $S$. aureus and $H$. influenzae pneumonia. However, in specific situations such as multilobar involvement, malnutrition, cavitation, Gram-negative necrotizing pneumonia, and/or isolation of $P$. aeruginosa or Acinetobacter spp., 14-21-day therapy should be initiated. Nonetheless, recent evidence has suggested that short treatment is as effective as longer treatment in VAP. Chastre and coworkers [48] evaluated 401 patients with VAP, 197 were randomized to receive short (8-day) treatment and 204 a long (15-day) course of antibiotic treatment. No differences were observed in the mortality rate $(18.8 \%$ vs. $17.2 \%)$ or in the recurrence of pulmonary infection ( $28.9 \%$ vs. $26 \%)$ on comparing the two groups of patients. However, those treated with a short course of antibiotics had significantly more antibiotic-free days $(13 \pm 7.4$ vs. $8.7 \pm 5.2$ days, $p<0.001)$. The possibility of providing adequate treatment with shorter courses of antibiotics will not only reduce health care costs but will also have favorable consequences on microbial ecology by reducing the selection pressure for resistance.

\section{Conclusion}

Nosocomial pneumonia is the leading cause of death among the hospital-acquired infections. Its incidence ranges from 4 to 50 cases per 1000 admissions in community hospitals and general medical wards. Aspiration of colonized pharyngeal secretions is considered the most important pathogenic mechanism of nosocomial pneumonia. Risk factors for development of nosocomial pneumonia include previous use of antibiotics, supine body position, stress ulcer prophylaxis, and duration of hospital admission. The diagnostic approach to these patients should start with the criteria of clinical suspicion discussed above and be followed by quantitative cultures of respiratory secretions obtained through tracheobronchial aspirate or bronchoscopic samples. Initial empiric antimicrobial therapy is based on the previous duration of hospital admission and the presence of risks factor for multi-drug resistant microorganisms.

\section{References}

1. Langer M, Cigada M, Mandelli M, Mosconi P, Tognoni G (1987) Early-onset pneumonia: a multicenter study in intensive care units. Intensive Care Med 13:342-346

2. Vincent JL, Bihari DJ, Suter PM, et al (1995) The prevalence of nosocomial infection in intensive care units in Europe. Results of the European Prevalence of Infection in Intensive Care (EPIC) Study. JAMA 274:639-644

3. Prod'hom G, Leuenberger P, Koerfer J, et al (1994) Nosocomial pneumonia in mechanically ventilated patients receiving antacid, ranitidine, or sucralfate as prophylaxis for stress ulcer. Ann Intern Med 120:653-662

4. Torres A, Aznar R, Gatell JM, et al (1990) Incidence, risk, and prognosis factors of nosocomial pneumonia in mechanically ventilated patients. Am Rev Respir Dis 142:523-528 
5. Sopena N, Sabriá M, and the Neunos Study group (2005) Multicenter study of hospitalacquired pneumonia in non-ICU patients. Chest 127:213-219

6. Langer $M$, Mosconi $P$, Cigada $M$, Mandelli $M$ (1989) Long-term respiratory support and risk of pneumonia in critically ill patients. Intensive Care Unit Group of Infection Control. Am Rev Respir Dis 140:302-305

7. Fagon JY, Chastre J, Domart Y, et al (1989) Nosocomial pneumonia in patients receiving continuous mechanical ventilation. Prospective analysis of 52 episodes with use of a protected specimen brush and quantitative culture techniques. Am Rev Respir Dis 139:877-884

8. Cook DJ, Walter SD, Cook RJ, et al (1998) Incidence of and risk factors for ventilator-associated pneumonia in critically ill patients. Ann Intern Med 129:433-440

9. Craven DE, Kunches LM, Kilinsky V, Lichtenberg DA, Make BJ, McCabe WR (1986) Risk factors for pneumonia and fatality in patients receiving mechanical ventilation. Am Rev Respir Dis 133:792-796

10. Kollef MH (1993) Ventilator-associated pneumonia: A multivariate analysis. JAMA 270:19651970

11. Lossos IS, Breuer R, Or R, et al (1995) Bacterial pneumonia in recipients of bone marrow transplantation. A five-year prospective study. Transplantation 60:672-678

12. Antonelli M, Moro ML, Capelli O, et al (1994) Risk factors for early-onset pneumonia in trauma patients. Chest 105:224-228

13. Cardeñosa Cendrero JA, Sole-Violan J, Bordes Benitez A, et al (1999) Role of different routes of tracheal colonization in the development of pneumonia in patients receiving mechanical ventilation. Chest 116:462-470

14. Johanson WG, Pierce AK, Sanford JP, Thomas GD (1972) Nosocomial respiratory infection with Gram-negative bacilli: the significance of colonization of the respiratory tract. Ann Intern Med 77:701-706

15. Estes RJ, Meduri GU (1995) The pathogenesis of ventilator-associated pneumonia: I. Mechanisms of bacterial transcolonization and airway inoculation. Intensive Care Med 21:365-383

16. Garrouste-Orgeas M, Chevret S, Arlet G, et al (1997) Oropharyngeal or gastric colonization and nosocomial pneumonia in adult intensive care unit patients. A prospective study based on genomic DNA analysis. Am J Respir Crit Care Med 156:1647-1655

17. Rello J, Sonora R, Jubert P, Artigas A, Rue M, Valles J (1996) Pneumonia in intubated patients: Role of respiratory airway care. Am J Respir Crit Care Med 154:111-115

18. Sirvent JM, Torres A, El-Ebiary M, Castro P, de Batlle J, Bonet A (1997) Protective effect of intravenously administered cefuroxime against nosocomial pneumonia in patients with structural coma. Am J Respir Crit Care Med 155:1729-1734

19. Rello J, Ausina V, Ricart M, Castella J, Prats G (1993) Impact of previous antimicrobial therapy on the etiology and outcome of ventilator-associated pneumonia. Chest 104:1230-1235

20. Trouillet JL, Chastre J, Vuagnat A, et al (1998) Ventilator-associated pneumonia caused by potentially drug-resistant bacteria. Am Rev Respir Dis 157:531 - 539

21. Torres A, Serra-Batlles J, Ros E, et al (1992) Pulmonary aspiration of gastric contents in patients receiving mechanical ventilation: the effect of body position. Ann Intern Med $116: 540-543$

22. Orozco-Levi M, Torres A, Ferrer M, et al (1995) Semirecumbent position protects from pulmonary aspiration but not completely from gastroesophageal reflux in mechanically ventilated patients. Am J Respir Crit Care Med 152:1387-1390

23. Drakulovic MB, Torres A, Bauer TT, Nicolas JM, Nogue S, Ferrer M (1999) Supine body position as a risk factor for nosocomial pneumonia in mechanically ventilated patients: a randomised trial. Lancet 354:1851-1858

24. Tryba M (1987) Risk of acute stress bleeding and nosocomial pneumonia in ventilated intensive care patients: Sucralfate versus antacids. Am J Med 83:117-124

25. Cook D, Guyatt G, Marshall J, et al (1998) A comparison of sucralfate and ranitidine for the prevention of upper gastrointestinal bleeding in patients requiring mechanical ventilation. Canadian Critical Care Trials Group. N Engl J Med 338:791 - 797

26. Messori A, Tripoli S, Vaiani $M$, et al (2003) Bleeding and pneumonia in intensive care patients given ranitidine and sucralfate for prevention of stress ulcer: meta-analysis of randomised controlled trials. BMJ 32:1103-1106

27. Fabregas N, Ewig S, Torres A, et al (1999) Clinical diagnosis of ventilator associated pneumo- 
nia revisited: comparative validation using immediate post-mortem lung biopsies. Thorax 54: $867-873$

28. Pugin J, Auckenthaler R, Mili N, Janssens JP, Lew PD, Suter P (1991) Diagnosis of ventilator associated pneumonia by bacteriologic analysis of bronchoscopic and non-bronchoscopic "blind" bronchoalveolar lavage fluid. Am Rev Respir Dis 143:1121-1129

29. Fartoukh M, Maitre B, Honore S, et al (2003) Diagnosing pneumonia during mechanical ventilation: The clinical pulmonary infection score revisited. Am J Respir Crit Care Med 168: $173-179$

30. Ioanas M, Ferrer M, Angrill J, Ferrer M, Torres A (2001) Microbial investigation on vetilatorassociated pneumonia. Eur Respir J 17:791-801

31. Valencia M, Torres A, Insausti J, et al (2003) Valor diagnóstico del cultivo cuantitativo del aspirado endotraqueal en la neumonía adquirida durante la ventilación mecánica. Estudio multicéntrico. Arch Bronconeumol 39:394-399

32. Torres A, Puig de la Bellacasa J, Xaubet A, et al (1989) Diagnostic value of quantitative cultures of bronchoalveolar lavage and telescoping plugged catheters in mechanically ventilated patients with bacterial pneumonia. Am Rev Respir Dis 140:306-310

33. Sanchez-Nieto JM, Torres A, Garcia-Cordoba F, et al (1998) Impact of invasive and noninvasive quantitative culture sampling on outcome of ventilator-associated pneumonia: a pilot study. Am J Respir Crit Care Med 157:371 - 376

34. Sole-Violan J, Fernandez JA, Benitez AB, Cardenosa Cendrero JA, Rodriguez de Castro F (2000) Impact of quantitative invasive diagnostic techniques in the management of outcome of mechanically ventilated patients with suspected pneumonia. Crit Care Med 28:2737-2741

35. Ruiz M, Torres A, Ewig $S$, et al (2000) Noninvasive versus invasive microbial investigation in ventilator-associated pneumonia. Am J Respir Crit Care Med 162:119-125

36. Fagon JY, Chastre J, Wolff M, et al (2000) Invasive and noninvasive strategies for management of suspected ventilator-associated pneumonia. Ann Intern Med 132:621-630

37. Torres A, Ewig S (2004) Diagnosing ventilator-associated pneumonia. N Engl J Med 350: $433-435$

38. Alvarez-Lerma F (1996) Modification of empiric antibiotic treatment in patients with pneumonia acquired in the intensive care unit. ICU-acquired Pneumonia Study Group. Intensive Care Med 22:387-94

39. Luna CM, Vujacich P, Niederman MS, et al (1997) Impact of BAL data on therapy and outcome of ventilator-associated pneumonia. Chest 111:676-687

40. Rello J, Diaz E (2003) Pneumonia in the intensive care unit. Crit Care Med 31:2544-2551

41. Kollef MH, Ward S (1998) The influence of mini-BAL cultures on patient outcomes: implications for the antibiotic management of ventilator-associated pneumonia. Chest 113:412-420

42. Rello J, Gallego M, Mariscal D, Sonora R, Valles J (1997) The value of routine microbiologic investigation in the diagnosis of ventilator-associated pneumonia. Am J Respir Crit Care Med 156:196-200

43. American Thoracic Society (1996) Hospital-acquired pneumonia in adults: Diagnosis, assessment of severity, initial antimicrobial therapy, and preventative strategies. A consensus statement. Am J Respir Crit Care Med 153:1711-1725

44. Ioanas $M$, Cavalcanti $M$, Ferrer $M$, et al (2003) Hospital-acquired pneumonia: coverage and treatment adequacy of current guidelines. Eur Respir J 22:876-882

45. Niederman M, Craven D (2005) Guidelines for the management of adults with hospitalacquired pneumonia, ventilator-associated pneumonia, and healthcare-associated pneumonia. Am J Respir Crit Care Med 171:388-416

46. Hilf M, Yu VL, Sharp J, Zuravleff JJ, Korvick JA, Muder RR (1989) Antibiotic therapy for Pseudomonas aeruginosa bacteremia: outcome correlations in a prospective study of 200 patients. Am J Med 87:540-546

47. Korvick JA, Bryan CS, Farber B, et al (1992) Prospective observational study of Klebsiella bacteremia in 230 patients: outcome for antibiotic combinations versus monotherapy. Antimicrob Agents Chemother 36:2639-2644

48. Chastre J, Wolff M, Fagon JY, et al (2003) Comparison of 8 vs 15 days of antibiotic therapy for ventilator-associated pneumonia in adults: a randomized trial. JAMA 290:2588-2598 